\title{
A Direct Comparison of Spatial Attention and Stimulus-Response Compatibility between Mice and Humans
}

\author{
Ulf H. Schnabel ${ }^{1}$, Tobias Van der Bij1 ${ }^{1}$, Pieter R. Roelfsema ${ }^{1,2,3}$, \\ and Jeannette A. M. Lorteije $\mathrm{L}^{1,2}$
}

\begin{abstract}
Mice are becoming an increasingly popular model for investigating the neural substrates of visual processing and higher cognitive functions. To validate the translation of mouse visual attention and sensorimotor processing to humans, we compared their performance in the same visual task. Mice and human participants judged the orientation of a grating presented on either the right or left side in the visual field. To induce shifts of spatial attention, we varied the stimulus probability on each side. As expected, human participants showed faster RTs and a higher accuracy for the side with a higher probability, a well-established effect of visual attention. The attentional effect was only present in mice when their response was slow. Although the task demanded a judgment of grating orientation, the accuracy of the mice was strongly affected by whether the side of the stimulus corresponded to the side of
\end{abstract}

\section{INTRODUCTION}

The mouse is an increasingly popular model to study visual processing and cognitive functions such as perceptual decision-making (Steinmetz, Zatka-Haas, Carandini, \& Harris, 2019; Carandini \& Churchland, 2013). Indeed, researchers studying the mouse can use a wide and unique range of genetic tools that allow investigation of causal relations between neural activity and behavioral responses (Guo et al., 2014; Sachidhanandam, Sreenivasan, Kyriakatos, Kremer, \& Petersen, 2013). Mice have a visual system that is in many ways similar to that of humans (Carandini \& Churchland, 2013; Katzner \& Weigelt, 2013), including a hierarchically organized system of visual areas (Wang \& Burkhalter, 2007). Neurons in the mouse primary visual cortex are tuned to a variety of features, including orientation, direction, and size (Self et al., 2014; Vaiceliunaite, Erisken, Franzen, Katzner, \& Busse, 2013; Adesnik, Bruns, Taniguchi, Huang, \& Scanziani, 2012; Niell \& Stryker, 2008), similar to neurons in the primary visual cortex area of primates (Van Den Bergh, Zhang, Arckens, \& Chino, 2010). However, there are also several

\footnotetext{
${ }^{1}$ Netherlands Institute for Neuroscience, ${ }^{2}$ University of Amsterdam, ${ }^{3}$ Vrije Universiteit, Amsterdam, The Netherlands

the behavioral response. This stimulus-response compatibility (Simon) effect was much weaker in humans and only significant for their fastest responses. Both species exhibited a speedaccuracy trade-off in their responses, because slower responses were more accurate than faster responses. We found that mice typically respond very fast, which contributes to the stronger stimulus-response compatibility and weaker attentional effects, which were only apparent in the trials with slowest responses. Humans responded slower and had stronger attentional effects, combined with a weak influence of stimulus-response compatibility, which was only apparent in trials with fast responses. We conclude that spatial attention and stimulus-response compatibility influence the responses of humans and mice but that strategy differences between species determine the dominance of these effects.

dissimilarities between mice and primates. For one, the mouse retina does not have a fovea, the spot on the retina with a high concentration of cones that is common in primates. Instead, the mouse retina has a more homogeneous distribution of photoreceptors with a lower density, which is comparable to the peripheral visual field in humans, although a nasal-to-temporal ganglion gradient does suggest slightly higher sampling of the binocular region (Bleckert, Schwartz, Turner, Rieke, \& Wong, 2014). Mice do not appear to perform frequent (Payne \& Raymond, 2017; Sakatani \& Isa, 2007) saccadic eye movements to move their fovea to a position of interest, whereas these eye movements occur frequently in primates. In this study, we investigated if and how differences between species influence performance in a task that induces shifts of visual attention in humans. We compared the influence of spatial attention, stimulus-response compatibility, and speed-accuracy trade-offs between the two species.

\section{Spatial Attention}

Spatial attention allows an organism to prioritize the processing of information at a certain location in space (Posner, 1980). Some psychophysical tasks induce endogenous shifts of attention with a central cue that summons 
attention to a location, such as an arrow (Herrero, Gieselmann, Sanayei, \& Thiele, 2013; Posner, 1980); others, with a peripheral cue presented at the location of interest, which shifts attention in a more automatic, exogenous manner (Wang, Chen, Yan, Zhaoping, \& Li, 2015; Arrington, Carr, Mayer, \& Rao, 2000). It is also possible to achieve an endogenous shift in attention by increasing the probability that a stimulus will appear at a particular location. These probability variations have been successfully used to cue attention in humans (Shomstein \& Yantis, 2004) and monkeys (Nandy, Nassi, \& Reynolds, 2017; Bushnell, Goldberg, \& Robinson, 1981). Shifts of spatial attention are associated with enhanced firing rates of cells with a receptive field at the attended location in the in the visual, parietal, and frontal cortices of monkeys (Nandy et al., 2017; Wang et al., 2015; Poort et al., 2012; Herrington \& Assad, 2009; Shomstein \& Behrmann, 2006; Reynolds \& Chelazzi, 2004; Bisley \& Goldberg, 2003; Schall \& Thompson, 1999; Gottlieb, Kusunoki, \& Goldberg, 1998; Roelfsema, Lamme, \& Spekreijse, 1998; Corbetta, Shulman, Miezin, \& Petersen, 1995; Schall \& Hanes, 1993; Bushnell et al., 1981) and humans (Self et al., 2016). These attention shifts can also be measured with neuroimaging methods in humans (Gandhi, Heeger, \& Boynton, 1999; Rosen et al., 1999; Somers, Dale, Seiffert, \& Tootell, 1999; Müller, Teder-Sälejärvi, \& Hillyard, 1998).

A recent study demonstrated that mice exhibit selective spatial attention in a go/no-go behavioral paradigm (Wang \& Krauzlis, 2018). In these experiments, head-restrained mice saw two orientated grating stimuli, and they had to detect when the orientation of one of the gratings changed. The animals' responses were faster and more accurate when a peripherally presented stimulus cued the location of the upcoming changed stimulus, compared to trials without a cue or trials in which a nonchanging stimulus was cued invalidly. Hence, spatial cueing causes attention shifts in mice. This result was confirmed in another mouse study using a go/no-go paradigm in which mice had to detect lowcontrast stimuli that could occur at one of two locations (Speed, Del Rosario, Mikail, \& Haider, 2020). Spatial attention has also been described in rats (Bushnell, 1995) and chickens (Sridharan, Ramamurthy, Schwarz, \& Knudsen, 2014), two other species that lack a fovea. Here, we will test the generality of attention shifts in mice to a task in which the animals have to discriminate between two stimuli, which were associated with different behavioral responses. Furthermore, we will directly compare the mouse results to those obtained in humans with the same paradigm. The use of two different behavioral responses instead of a go/no-go paradigm is advantageous because it will also allow us to also measure stimulus-response compatibility effects.

\section{Stimulus-Response Compatibility (Simon Effect)}

Simon (1969) found that responses of human participants to an auditory stimulus were faster if the side of presentation of the stimulus corresponded to the side of the response than on trials in which these sides did not correspond. This effect was replicated in the visual domain (Craft \& Simon, 1970; see Lu \& Proctor, 1995, for a review). Imagine a task in which a button on the left should be pressed when a red stimulus is presented and a button on the right for green stimuli. If a red stimulus, instructing a left button press, is presented on the left side, the side of the stimulus is compatible with the side of the response and RTs are shorter than if the red stimulus is presented on the right. Influential models aiming to explain the Simon effect pose that there are two competing responses between which a selection should be made (De Jong, Liang, \& Lauber, 1994; for a recent review, see Salzer, de Hollander, \& Forstmann, 2017) and which may involve inhibition of the incorrect response (Ridderinkhof, Scheres, Oosterlaan, \& Sergeant, 2005). The temporal, "what," stream is thought to process the color and to activate the appropriate response. However, the irrelevant spatial layout is thought to be processed in the faster dorsal, "where," stream and activates the response toward the corresponding side. An error will be made if this fast response is not suppressed in trials demanding a response toward the side opposite to the stimulus. This competition between processing streams can explain the longer RTs on incongruent trials. Mice have a hierarchical visual cortex in which dorsal- and ventral-like pathways can be distinguished (Wang, Gao, \& Burkhalter, 2011), and they may therefore also exhibit a Simon effect. Indeed, a previous study (Courtière, Hardouin, Burle, Vidal, \& Hasbroucq, 2007) demonstrated a Simon effect in freely moving rats using auditory stimuli. However, although rats and mice are close relatives, it would be useful to extend these results to mice and to the visual domain. Furthermore, we aimed to establish a paradigm that permits head fixation, because it would permit many of the techniques by which neural pathways in mice can be explored.

In the current study, we instructed humans and trained mice to perform an orientation discrimination task. We cued the participants' attention to one of two locations by varying the probability of the stimuli. We expect to see a higher accuracy or faster responses at the side with the higher probability compared to the other side with the lower probability. The lateralized stimuli and responses in the task also allowed us to investigate stimulus-response compatibility effects in both species. Establishing a paradigm for the Simon effect and spatial attention in mice would allow researchers to investigate the underlying neuronal mechanisms using the many novel techniques available in this species, like the optogenetic manipulation of neural activity (Guo et al., 2014).

\section{METHODS}

\section{Experiments with Mice}

\section{Task and Stimuli}

We aimed to cue attention in an orientation discrimination task by manipulating the likelihood of stimulus appearance 
at one of two locations on the screen (Figure 1). The mice were first trained in simple versions of the orientation discrimination task. We trained them to indicate the orientation of a full-screen sinusoidal grating of $80 \%$ contrast, a spatial frequency of 0.02 cycles/degree, and an orientation of either $0^{\circ}$ or $90^{\circ}$ by licking the right or left side of a lick spout with two outlets, respectively (Figure 1A). The gratings were presented for $1.5 \mathrm{sec}$, and if the mouse did not respond within this period, the grating disappeared and the trial was aborted. The mouse received a drop of baby formula or water as a reward after every correct trial. Correct trials ended with an intertrial interval of 4-6 sec, and this interval was extended by an additional $5 \mathrm{sec}$ in case of an error.

Once the animals consistently performed this task above $75 \%$ accuracy, the size of the grating was gradually reduced to $40^{\circ}$. Next, across training sessions, we gradually moved the gratings toward the left and right of the screen until they reached their final position, at an eccentricity of $30^{\circ}$ (Figure 1B). Of the eight mice, only four learned the final version of the task, and the data of these four mice were included in our analysis.

In the final version of the task, we aimed to direct spatial attention of the mice to one of two possible stimulus positions by varying the probability in a block-wise manner. A block of trials started with 10 trials where the gratings appeared on one side of the screen. After these initial "cueing" trials, we presented stimuli at that "cued" location in $80 \%$ of trials (Figure 1B) and at the other, "uncued" location in $20 \%$ of trials. After a block of 32 trials, a new block started by cueing the other location with an initial set of 10 trials followed by test trials. We used blocks with 32 trials, so that the two cueing conditions could be sufficiently interleaved. We included all sessions of the final version of the tasks in the analysis.

\section{Surgery and Training}

The experimental procedures complied with the National Institutes of Health Guide for Care and Use of Laboratory Animals, and the protocol was approved by the ethics committee of the Royal Netherlands Academy of Arts and Sciences. We used eight C57BL/6J male mice aged between 2 (at the start) and 12 (at the end) months, which were housed solitarily. All mice were implanted with a head post under $2 \%$ isoflurane anesthesia and antiseptic conditions. The head post allowed head fixation during behavioral testing. During the surgery, body temperature was maintained at $37^{\circ} \mathrm{C}$ with a heating pad. We applied Xylocaine as local analgesia and also gave systemic analgesia (Metacam, subcutaneously, $2 \mathrm{mg} / \mathrm{kg}$ ). After 5 days of recovery from surgery, animals were habituated to head fixation in sessions spread out over several days. During the first sessions, the animals' head was only briefly fixated (1-3 min), and the period of head restraint was then gradually increased to 10-15 min. Animals were habituated in 3-10 sessions. We placed a half-tube over the animals during head fixation, which restricted their body movements and increased the comfort of animals because they like to stay in small compartments. Once

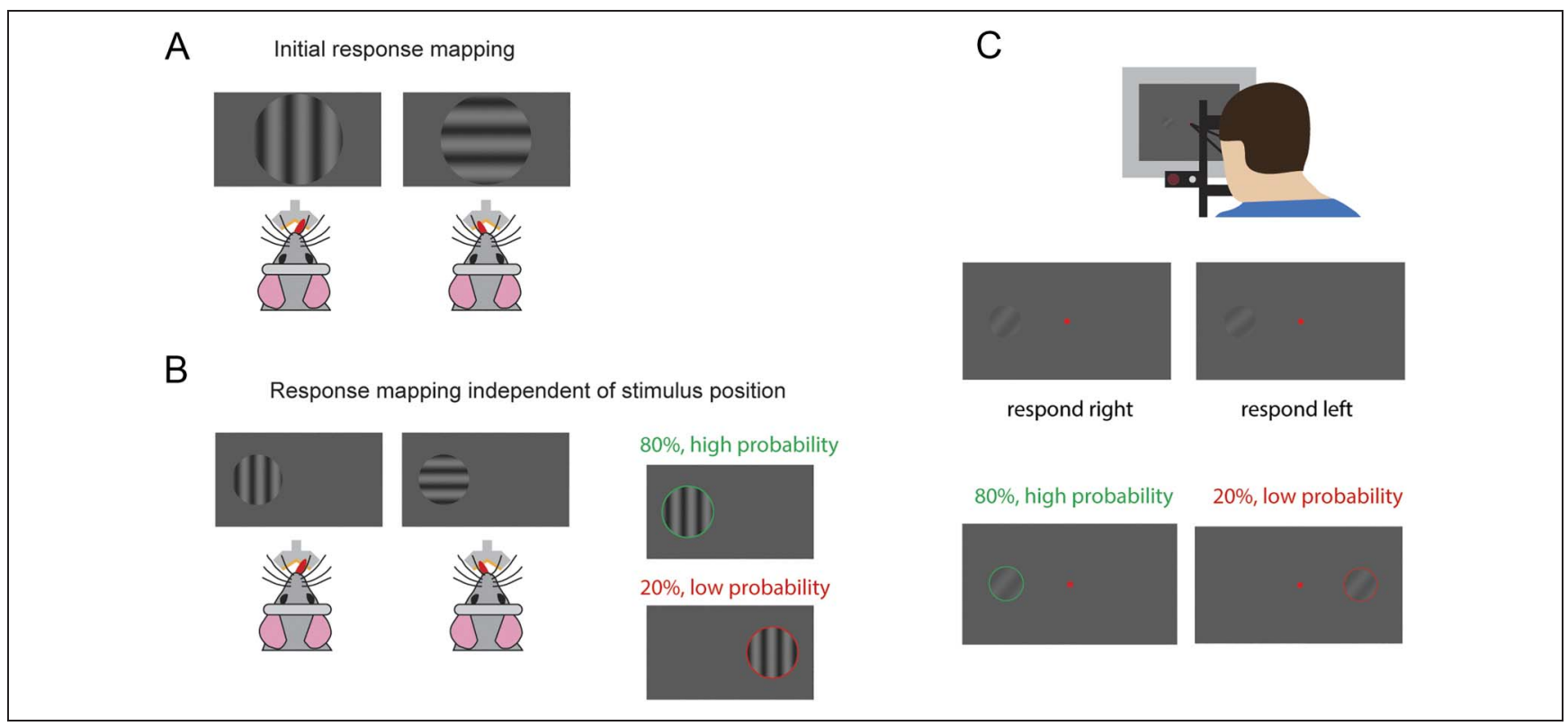

Figure 1. Orientation discrimination task. (A) The mice were trained to report the orientation of a sinusoidal grating presented on a screen in front of them. They indicated their choice by licking either the left or right side of a lick spout with two outlets. (B) In the final version of the task, the animals reported the orientation of the grating by licking left or right, regardless of stimulus position. During a block of trials, $80 \%$ of stimuli were presented at the high-probability location, whereas $20 \%$ were presented on the opposite side of the screen. (C) Human participants performed the same paradigm with modified stimuli. They responded by pressing a key on either side of a keyboard with their left or right index finger. Note that the difference between orientations is smaller and stimulus contrast is lower than that for the mice. 
the mice were habituated to this setup, they were placed on a regime with controlled fluid uptake and trained on the behavioral task. The animals minimally drank $25 \mathrm{ml} / \mathrm{kg}$ per day; otherwise, additional liquid was provided within the home cage. During the controlled fluid uptake regime, the animals were weighed daily and their general appearance was inspected. We gave the animals ad lib water during weekends and during the periods that they were not trained. The mice did not exhibit any physical or behavioral signs of compromised welfare because of the controlled fluid uptake protocol.

\section{Setup}

The head-fixed mice were positioned at a distance of $13.6 \mathrm{~cm}$ from a 21 -in. LCD screen (DELL 059DJP) driven at a resolution of $1280 \times 720$ pixels at $60 \mathrm{~Hz}$. The maximum luminance of the screen was set to $30 \mathrm{~cd} / \mathrm{m}^{2}$. We used custom-made software using Cogent Graphics (developed by John Romaya at the LON at the Wellcome Department of Imaging Neuroscience) running in MATLAB (The MathWorks, Inc.) for stimulus presentation and behavioral monitoring. We recorded the animals' licks by measuring the influence of the proximity of the tongue on the capacitance of metal plates on a custom-made double-sided lick spout (Figure 1A) using an Arduino Uno. The double lick spout distinguished between licking responses to a leftand-right lick port.

\section{Analysis}

We combined trials across all sessions (average of 15.3 per mouse) of each mouse and analyzed 16,995 (average of 4248 per mouse) hit and error trials with an RT between 150 and $800 \mathrm{msec}$ after stimulus onset. Trials with faster $(n=948,5.2 \%)$ or slower $(n=359,2.0 \%)$ RTs were excluded because we assumed that licks before $150 \mathrm{msec}$ were because of random licking. Fast responses were more frequent at the beginning of a session when animals were eager to work. Very slow responses, which are indicative of loss of motivation or distraction, were more frequent toward the end of a session. We ran two-sample $t$ tests on each mouse's individual data. We analyzed the RT and accuracy data across the group of animals with a repeated-measures ANOVA. Our initial analysis did not reveal an overall effect of attention. To confirm this surprising negative finding, we also performed a less conservative paired $t$ test across mice, as stronger indicator of a true negative result.

\section{Experiments with Human Participants}

\section{Participants}

We tested seven participants with a mean age of $23( \pm 2.3)$ years who reported normal or corrected-to-normal visual acuity. They were paid for their participation. Four were female and three were male, and all but one participant were right-handed. The ethics committee of the Faculty of Social and Behavioural Sciences at the University of Amsterdam approved the experiments. Participants were instructed about the task, and informed consent was obtained before the start of the experiment. The data from all participants were included in the analysis.

\section{Visual Stimuli and Task}

Human participants performed a task that was almost identical to the one used in mice. After instructing participants about the task and explaining the probability manipulation, we presented sinusoidal gratings of two orientations on the left or right side of a fixation point and asked them to indicate the orientation of the grating by pressing a button on the left or right side of a keyboard. In the version for human participants, the gratings had an orientation of either $40^{\circ}$ or $50^{\circ}$ and a contrast of $20 \%$. We presented stimuli with a lower contrast and a smaller orientation difference than for the mice, to avoid ceiling performance. The participants had to fixate on a black fixation point for $300 \mathrm{msec}$ to initiate a trial. The stimulus, a $5^{\circ}$ sinusoidal gratings with a spatial frequency of 0.3 cycles/degree, was then presented at an eccentricity of $10^{\circ}$, to the left or right of the fixation point, for a maximum duration of $1.5 \mathrm{sec}$ or until the participants pressed the keyboard. The background luminance of the screen was $7.7 \mathrm{~cd} / \mathrm{m}^{2}$. If the participants broke fixation (i.e., left a fixation window with a size of $3^{\circ} \times 3^{\circ}$ centered on the fixation point) before the keyboard response, the trial was aborted and participants saw a message stating they failed to fixate. Trials were followed by a variable intertrial interval of 2.6-2.8 sec, during which there was no fixation requirement.

Each participant performed one experimental session of 1200 trials, which followed an initial practice session with two blocks of 50 trials. Participants reported the orientation of the grating by pressing the " $\mathrm{z}$ " or "/" button on a keyboard with their left or right index finger. The stimuli were presented in blocks of 200 trials. The first 50 trials in each block were cueing trials during which stimuli were presented at one of the two possible locations only. These cueing trials were not included in the analysis. After the cueing trials, 150 experimental trials followed, with 80\% of the trials with a stimulus at the cued location and the other 20\% at the noncued location (Figure 1C). We gave feedback after every trial. The fixation dot turned red during the intertrial interval after errors and green after correct responses.

\section{Setup}

The participants sat at a distance of $57 \mathrm{~cm}$ from a CRT screen (Figure 1C), with their head supported by a chin rest. The luminance of the screen was set to range between 0.005 and $15.4 \mathrm{~cd} / \mathrm{m}^{2}$. Stimuli were controlled by a 
Windows computer running MATLAB (The MathWorks, Inc.) and Cogent Graphics. We monitored the eye position using an Eyelink T1000 (SR Research Ltd.) system sampling at $1000 \mathrm{~Hz}$, which was calibrated before the start of the session.

\section{Analysis}

We analyzed only hit and error trials with an RT between 150 and 1500 msec and excluded trials outside this range. These exclusion criteria differed from those for the mouse data because the overall RTs of human participants were longer (see below). Just as for the mice, we performed two-sample $t$ tests for the data of individual subjects and used an ANOVA to analyze the results across participants.

\section{Analysis Steps Applied to the Data of Mice and Humans}

\section{Analysis of the Speed-Accuracy Trade-off}

To obtain insight into a possible trade-off between speed and accuracy, we sorted all trials of individual subjects in 11 bins, based on the $z$ score of the RT distribution. We determined the subject's accuracy in each bin. We used a bootstrapping procedure to estimate the variance of the accuracy in each bin. We resampled the responses (correct, error) 1000 times with replacement and calculated the average accuracy. These values were used to generate the Bonferroni-corrected (nine comparisons) significance threshold at an alpha of 5\% for comparing each bin to its neighbor.

\section{Splitting Trials in Fast and Slow According to RT}

To compare effects on trials where subjects responded very quickly to those where they took more time, we split trials according to the $z$ score of the RT distribution within each subject. In humans, trials with a $z$ score $\leq 1$ (this is the faster $15.9 \%$ of the RT distribution) were marked as fast; and the remaining trials, as slow. In mice, trials with a $z$ score $\leq 0$ were marked as fast, whereas trials with $0 \leq z$ score $\leq 1.5$ were marked as slow. We excluded trials with a $z$ score $>1.5$ because of the very low accuracy.

\section{Quantification of Stimulus-Response Compatibility (Simon Effect) and Attention}

Although subjects could ignore the location (left or right) of the stimulus because they had to only report stimulus orientation, the button press occurred at the same side as the stimulus on half the trials (compatible) and at the other side on the remaining trials (incompatible). To investigate whether the compatibility of stimulus and response affects the accuracy of the responses, we used the following formula to measure this potential effect:

$$
\begin{aligned}
& \text { Compatibility effect } \\
& \quad=\text { Accuracy }_{\text {compatible }}-\text { Accuracy }_{\text {incompatible }}
\end{aligned}
$$

The compatibility effect is positive if subject's accuracy is higher on trials demanding a response on the same side as the stimulus. We calculated a similar measure, called attention effect, that compares the accuracy of validly cued stimuli to the accuracy of invalidly cued stimuli.

$$
\begin{aligned}
& \text { Attention effect } \\
& \quad=\text { Accuracy }_{\text {high }} \text { probability }- \text { Accuracy }_{\text {low probability }}
\end{aligned}
$$

\section{RESULTS}

Seven humans and four mice reported the orientation of a grating patch. This patch could be presented either on the left or right side of the screen. In a block of trials, stimuli would appear on one side with a high probability (80\% of trials) and on the other side with a low probability (20\%). Across blocks, the high- and low-probability sides changed. Thus, we attempted to endogenously cue spatial attention to one location in each block of trials.

We introduced small differences between the stimuli for humans and mice, attempting to correct for the higher visual acuity of humans and to make the task difficulty more similar. For human participants, we used a higher spatial frequency ( 0.3 vs. 0.02 cycles/degree), lowered contrast (20\% vs. $80 \%)$, and a smaller orientation difference $\left(10^{\circ}\right.$ vs. $\left.90^{\circ}\right)$ (Figure 1$)$. The mice were head-fixed, and the human participants placed their chin on a chin rest and they had to maintain gaze at a fixation point during the presentation of the grating. Another important difference was that we rewarded mice with a drop of water upon every correct trial, whereas humans received a monetary reward for their participation that did not depend on their performance.

Both mice and humans performed the lateralized orientation discrimination task with accuracies far above chance level (Figure 2A, left). The average accuracy of the four mice was $72 \pm 7.8 \%$ (mean $\pm S E M$, significantly higher than chance level, $t$ test, $t(3)=5.6, p=.01$ ), and the accuracy of the seven human participants was $86 \pm 1 \%, t(6)=28.00$, $p<.001$. Although the mouse paradigm was much easier, the accuracy of mice was lower than that of human participants (two-sided $t$ test, $t(9)=4.32, p<.01$ ). Interestingly, the average RTs of mice (332 $\pm 57 \mathrm{msec})$ were much shorter than those of humans $(628 \pm 26 \mathrm{msec}), t(9)=$ $7.74, p<.001$ (Figure 2A, right). Part of the difference in RT between species is presumably because of a different type of response; the mice licked a spout, and the humans pressed a key on a keyboard. 
Figure 2. Accuracy and RTs of humans and mice. (A) Accuracy and RT in the orientation discrimination task. (Left) Humans performed the task at a higher accuracy than mice, but both species were able to perform the task well above chance level (50\%). (Right) The RTs of humans were almost twice as large as the RTs of mice. Errors bars indicate SEM. (B) Accuracy as a function of RT. Both species exhibited a speedaccuracy trade-off. Faster responses were of lower accuracy than the ones in the middle of the RT distribution. The accuracy of very slow responses was also low, which suggests that the human participants and mice might have been distracted on a fraction of the trials. Errors bars indicate bootstrapped 99\% confidence intervals.

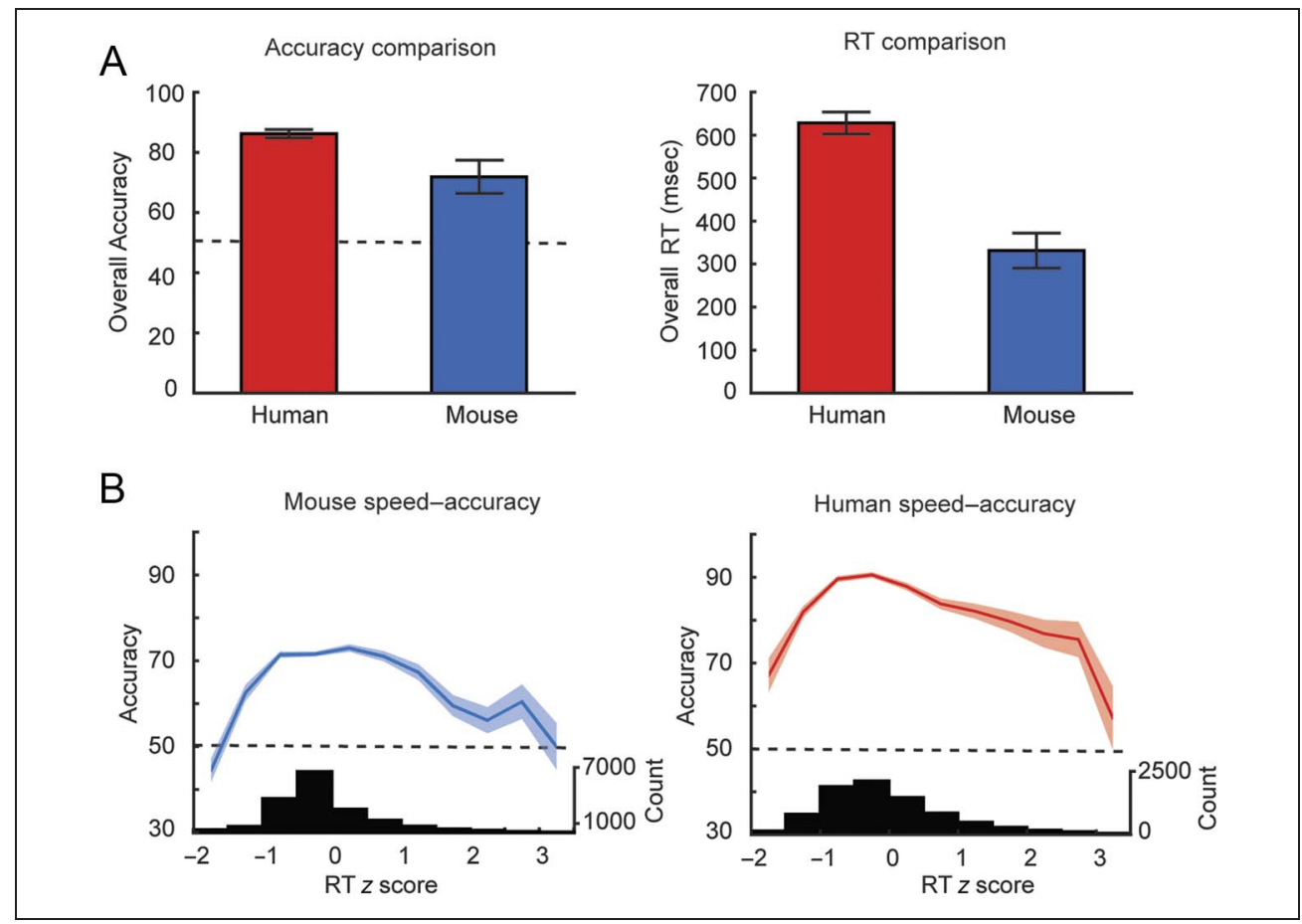

\section{Speed-Accuracy Trade-off}

These differences in accuracy and RT between species prompted us to investigate whether the mice traded speed against accuracy in a different way than the humans. We therefore $z$-scored RTs in each subject and determined the accuracy in different bins of the RT distribution (Figure 2B). To test the influence of response speed on accuracy, we used a bootstrapping procedure (see Methods). In both species, responses in the fastest bin $(z$ score of RT between -2 and -1.5 ) were less accurate than responses in the second fastest bin ( $z$ score between -1.5 and -1 ), which were in turn significantly less accurate than responses in the third bin $(z$ score between -1 and -0.5 ; bootstrap test, all $p s<.05$; see Methods). Hence, we obtained evidence for a trade-off between speed and accuracy in both species.

\section{Spatial Attention}

We next investigated the possible effects of spatial attention. By repeatedly presenting the stimuli at one side of the screen, we expected the subjects to allocate attention to that side. Spatial attention is expected to lead to shorter RTs at the high-probability location and longer RTs at the low-probability location.

The RTs of the human participants depended on stimulus probability (Figure $3 \mathrm{~A}$ and $\mathrm{B}$ ) and were shorter for the high-probability location ( $622 \mathrm{msec}, S E M=25 \mathrm{msec}$ ) than the low-probability location (657 $\pm 26 \mathrm{msec}$; ANOVA, $F(1,6)=91.5, p<.001)$. The probability effect on RT was present in each of seven participants (Figure 4B; $t$ tests, all $p s<.05)$. Cueing also influenced the accuracy (Figure 3C), which was higher at the cued location $(87 \pm 1 \%)$ than at the noncued location ( $81 \pm 2 \%$; ANOVA, $F(1,6)=17.1$, $p<.05)$. We additionally tested if this influence on accuracy is dependent on the speed of responses (Figure 5A). The attention effect (Accuracy high probability $_{\text {- Accuracy }}$ low probability; see Methods) was only $0.6 \pm 0.4 \%$ on trials with shorter RTs ( $z$ score $\leq-1$; the fastest $15.9 \%$ of the RT distribution) and did not differ significantly from $0, t(6)=$ $0.19, p=.85$, whereas it was $6.25 \pm 1.4 \%$ (paired $t$ test, $t(6)=4.6, p<.01)$ on the slower trials. This influence of RT on the magnitude of the attention effect was significant, $t(6)=2.28, p=.031$. Hence, the subjects' attention was attracted to the high-probability location, but the attentional effect was absent on trials with fast responses.

We examined the responses of mice for a similar cueing effect. Surprisingly, RTs of mice did not depend on stimulus probability (Figure 3B). The average RT was $331 \pm$ $32 \mathrm{msec}$ at the cued location and $334 \pm 38 \mathrm{msec}$ at the noncued location (ANOVA, $F(1,3)=0.09, p=.78$ ). We also used a more sensitive paired $t$ test but did not find a significant effect of cueing either, $t(3)=0.63, p=.57$, and such an effect was also absent from the within-animal comparisons (Figure 4C, $t$ tests, all $p s>$.05). Only Mouse 1 showed a trend toward faster responses to the high-probability location, but the effect failed to reach significance, $t(965)=$ $1.84, p=.06$. The accuracy (Figure $3 \mathrm{C}$ ) at the cued location $(71 \pm 5 \%)$ also did not differ significantly from the accuracy at the noncued location ( $72 \pm 4 \%$; ANOVA, $F(1$, $3)=1.0, p=.4)$, and this negative finding was confirmed with the more sensitive paired $t$ test, $t(3)=1.62, p=.20$, and with an analysis of data of individual mice (Figure 4D, $t$ tests, all $p s>.05)$. We then tested if the attention effect might differ between slow and fast trials (Figure 5B), just 
as was the case for the human participants. On faster trials $(z$ score $\leq 0$, which corresponded to the $66 \%$ of trials with the shortest RTs, given the skewness of the RT distribution; see Figure 5B), the attention effect was $0.7 \pm$ $1 \%$ and not significant, $t(3)=0.074, p=.95$. However, we did observe a weak but significant attention effect of $2.4 \pm 0.6 \%$ in the trials with longer RTs $(0 \leq z$ score $\leq$ $1.5 ; 27 \%$ of trials, $t(3)=4.53, p=.02)$. Here, we excluded trials with very long RTs $(z$ score $>1.5)$ because accuracy in these trials was very low, which was probably because of the mice being less motivated or aroused. If we included all trials with $z$ score $>0$, the attention effect was also significant $(p=.03)$. These results suggest that spatial cueing causes a weak attention effect, but only on trials with longer RTs.

The blocks of trials in humans had 200 trials and were longer than the blocks in mice, with only 32 trials. To investigate if this difference may have played a role, we tested whether the effect of attention is visible if we only analyze the first 32 trials of each block from the human trials. In this restricted analysis, cueing influenced the RTs, which were shorter at the high-probability location (622 msec, SEM = $25 \mathrm{msec}$ ) than at the low-probability location (657 \pm 26 msec; paired $t$ test, $t=5.79, p=.001)$. Hence, the cueing effect in humans also occurred during the first trials of the block.

\section{Stimulus-Response Compatibility Effect (Simon Effect)}

We next examined the response compatibility effects. The location of the stimulus was irrelevant for the orientation judgment, but in stimulus-response compatible trials, the subjects had to respond to the same side as where the stimulus was presented, whereas in incompatible trials, they had to respond to the other side. In previous studies, stimulus-response compatibility led to faster responses in human participants (Simon, 1969).

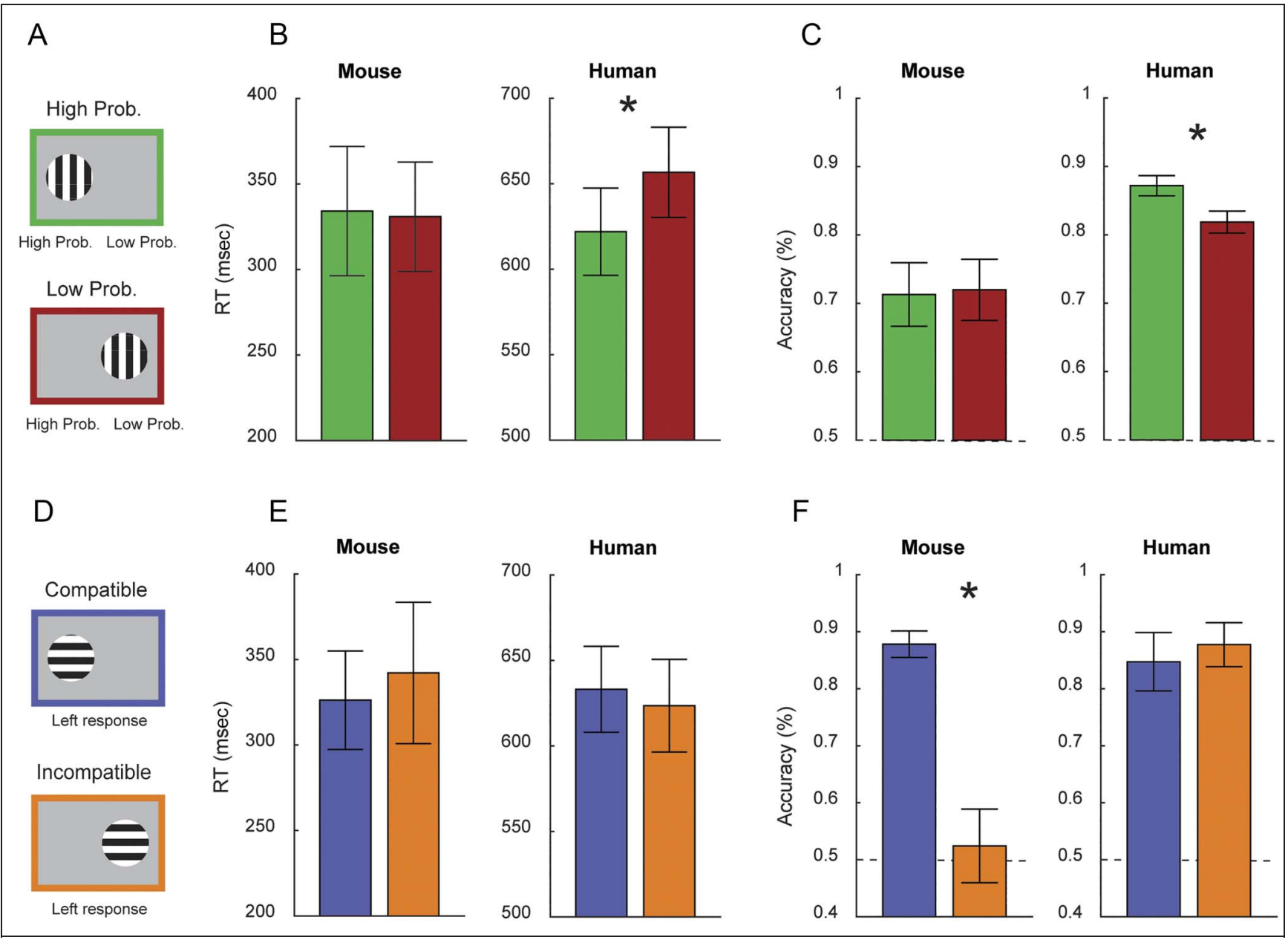

Figure 3. Influence of presentation probability and stimulus-response compatibility. (A) The probability of stimulus presentation was high at one location and lower at the other. (B) RTs at the high-probability location (green bars) and at the low-probability location (red bars). Humans responded significantly faster to stimuli at the high-probability location, whereas there was no overall effect for mice. (C) Accuracy of humans and mice. For humans, the accuracy was higher at the high-probability location (chance level is 50\%). (D) Responses on trials in which the stimulus and response were compatible (incompatible) are shown in blue (orange). (E) RTs as a function of response compatibility. The RT of neither humans nor mice was affected by stimulus-response compatibility. (F) Accuracy at the response compatible and incompatible locations. The accuracy of mice was strongly affected by stimulus-response compatibility, whereas there was no overall effect in humans. Prob. = probability. 


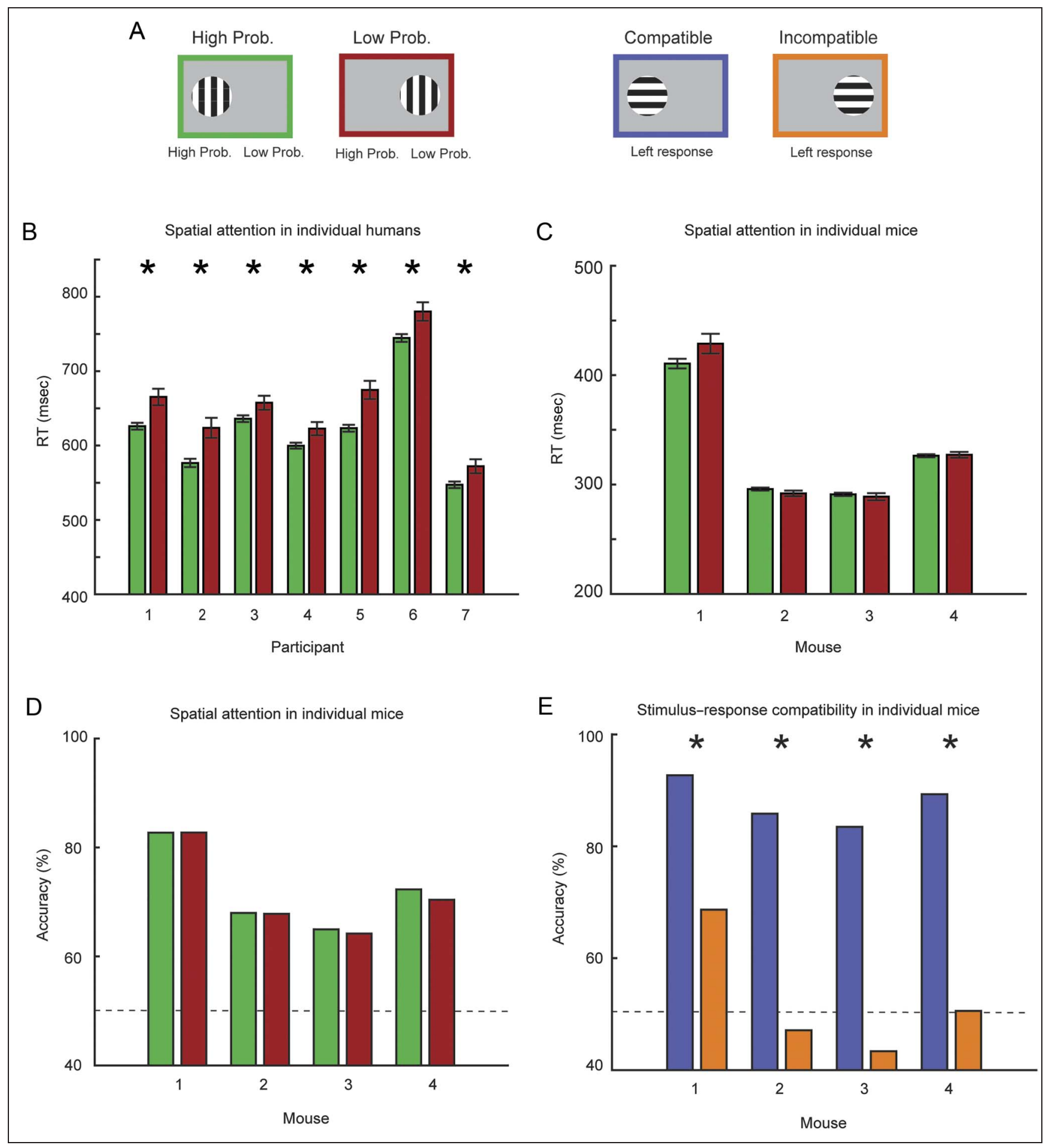

Figure 4. RT and accuracy of individual participants and mice. (A) Stimuli were presented at the high-probability (green) or low-probability (red) location. They were shown at the response-compatible (blue) or response-incompatible (orange) side. (B) The probability of presentation had a significant effect on the RT of every human participant. (C) Overall, there was no significant effect of presentation probability on RT in any of the mice. (D) Presentation probability also had no influence on the accuracy of mice. (E) The compatibility between stimulus and response strongly influenced the accuracy of all mice. In all panels, errors bars indicate SEM. Prob. = probability.

Human participants responded with almost the same RT (Figure 3D and E) on compatible $(624 \pm 27 \mathrm{msec})$ and incompatible $(633 \pm 26 \mathrm{msec})$ trials, and the effect of stimulus-response compatibility on RT was not significant (ANOVA, $F(1,6)=1.3, p=.30$ ). There was also no effect of stimulus-response compatibility on accuracy. The accuracy (Figure 3F) on compatible $(88 \pm 2 \%)$ and incompatible $(85 \pm 2 \%)$ trials was very similar. It has been shown that the Simon effect is stronger if the RT is short (De Jong et al., 1994). We therefore also calculated the compatibility 
Figure 5. Attention and response compatibility effects for trials with short and long RTs

(A) The influence of attention on the accuracy of human responses was not present on trials with short RTs. The panel on the lower right shows the $z$ scores of the RTs of trials with short (dark green) and long (light green) RTs. (B) Mice exhibit an effect of attention on trials with long RTs (dark blue) but not on trials with shorter RTs (light blue). Note that the $2.4 \%$ accuracy difference is smaller than that in humans $(6.25 \%$ accuracy) visible in A. (C) The stimulus-response compatibility effect in humans was only present for trials with short RTs. (D) Mice exhibit a strong effect of stimulus-response compatibility in all trials. In all panels, errors bars indicate SEM.

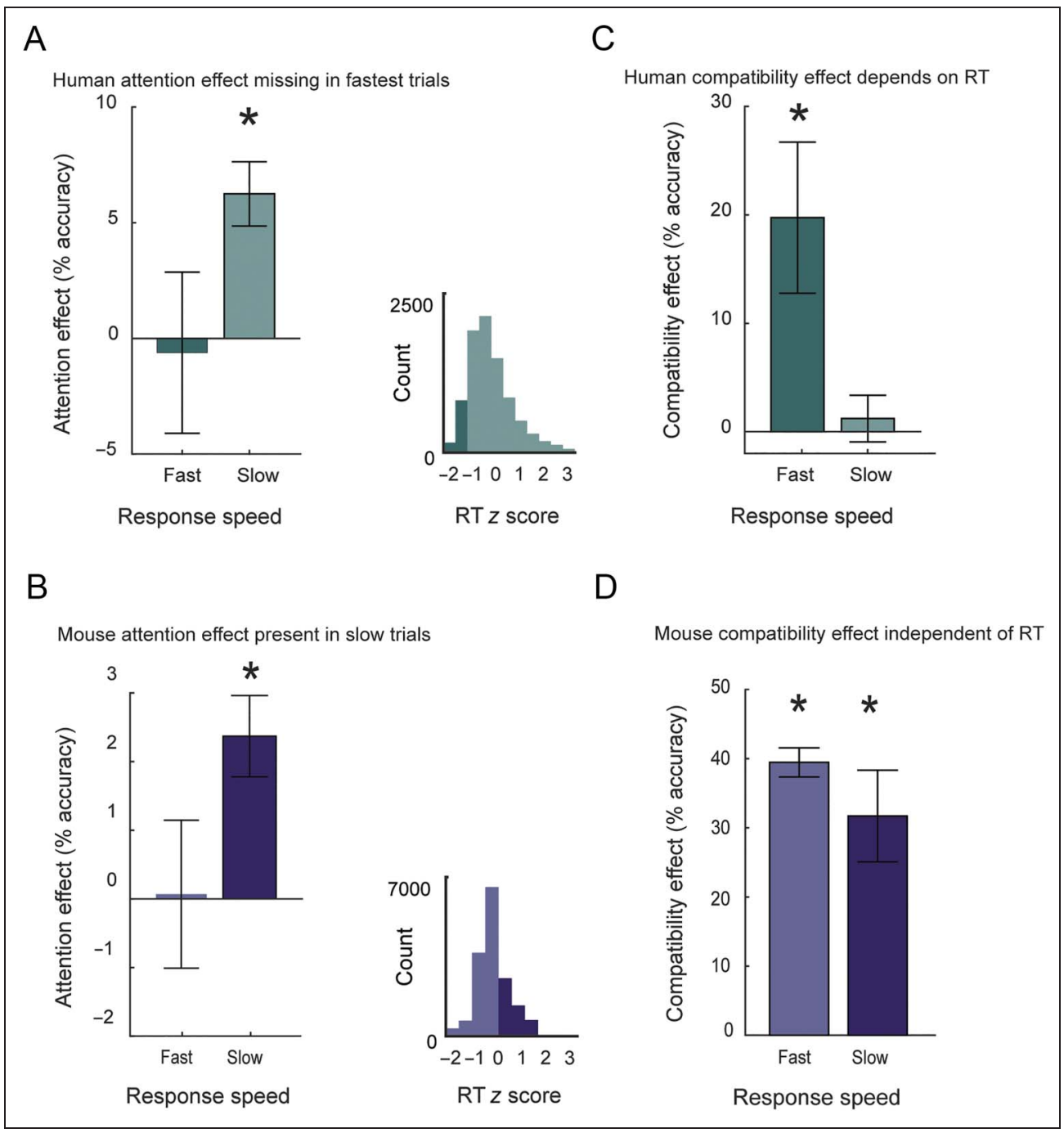

effect (Accuracy compatible $_{\text {- Accuracy }}$ incompatible) for trials with a shorter RTs (see above) and compared it to the effect for trials with longer RTs (Figure 5C). The compatibility effect for the fast responses was $20 \pm 7 \%$ ( $t$ test, $t(6)=3.1, p=.022)$. On trials with longer RTs, the effect was $1 \pm 2 \%$, which was not significant ( $t$ test, $t(6)=0.6$, $p=.56)$. Hence, human participants also exhibited a stimulus-response compatibility effect, but only on trials with a short RT.

Next, we examined the effect of stimulus-response compatibility in mice. Mouse RTs (Figure 3E) were slightly shorter on compatible $(326 \pm 29 \mathrm{msec})$ than on incompatible $(342 \pm 41 \mathrm{msec})$ trials, but this effect failed to reach significance (ANOVA, $F(1,3)=1.6, p=.30$ ). However, we did observe a strong effect of stimulus-response compatibility on the accuracy (Figure 3F). The accuracy was $88 \pm 2 \%$ on compatible trials but only $52 \pm 6 \%$ on incompatible trials (ANOVA, $F(1,3)=119.9, p=.002$ ), an effect that was reliable in each animal (Figure $4 \mathrm{E}$, all $p s<.001)$. These results revealed a very strong effect of stimulus-response compatibility in mice, indicating that the animals had difficulties in selecting a response opposite to the side of the stimulus. The stimulus-response compatibility effect was present on both fast and slow trials (see above; Figure 5D). Specifically, the effect was $40 \pm 2 \%$ in fast trials, $t(3)=22, p<.001$, and $30 \pm 7 \%$ in slow trials, $t(3)=11.5, p=.012$, and the difference in the magnitude of the effect did not differ significantly between slow and fast trials, $t(3)=1.59, p=.21$.

The mice were typically biased to responding toward one side, which was their preferred side. Across the four mice, this preference did not differ significantly between compatible and incompatible trials (59\% vs. $75 \%, p=$ .091). However, the probability that the mouse did not respond during a trial was higher for incompatible trials (24\%) than for compatible trials (9\%; paired $t$ test: $t(3)=$ 5.41, $p=.012)$.

\section{DISCUSSION}

We found that human participants responded faster and more accurately at the location where the stimulus was likely to appear, in accordance with a shift attention to the likely 
stimulus location (Nandy et al., 2017; Shomstein \& Yantis, 2004; Bushnell et al., 1981; Posner, 1980). They were also affected by stimulus-response compatibility, but only on trials with the fastest RTs. In mice, however, the dominating influence on performance was a stimulus-response compatibility effect, and we only observed a weak attentional effect on slow trials as if attention plays a much more pronounced role in the visual system of humans than in that of mice. In addition, our results suggest that mice may have been unable to suppress their responses long enough for the effects of spatial attention to take effect in most trials.

\section{Spatial Attention}

It is of interest that every human participant exhibited an effect of stimulus probability but that such an influence was absent in the overall results of the mice, although they performed more than three times the number of trials of the human participants (Figure $4 \mathrm{~B}$ and $\mathrm{C}$ ). The difference between species appeared to be related to the RTs of both species, which were typically short for mice and longer for humans. Indeed, the effect of spatial attention on accuracy in humans only occurred in trials with longer RTs and not in trials with the shortest RTs (Figure 5A). We found the same pattern of results in mice (Figure 5B) except that their typical RT was too short for an effect of spatial attention. The strong response compatibility effect in mice may have masked part of the attention effect on trials with short RTs. We note, however, that the stimulus-response compatibility effect was also present in mice on the trials with longer RTs, implying that the effects of response compatibility and attention can coexist. Another factor contributing to the differences in strength of attentional effects in mice and humans may have been the number of cuing trials before the side of stimulus presentation was manipulated, as well as the overall length of blocks. Humans were exposed to more cuing trials and performed much longer overall blocks than mice. This may have caused a stronger shift of attention to the high probability side in humans than in mice and could contribute to the stronger effects of spatial attention in humans, although the attentional effect was also present in humans when we only analyzed the first trials of a block.

Our findings confirm that mice can shift spatial attention but suggest that they failed to do so on most of the trials in our paradigm. Indeed, two recent studies also provided evidence that mice shift spatial attention (Speed et al., 2020; Wang \& Krauzlis, 2018). Both studies used a go/nogo paradigm in which the mice detected a stimulus or change of a stimulus so that they could not examine stimulus-response compatibility effects. In the paradigm of Wang and Krauzlis, the mice responded to a change in the orientation of one of two gratings and did not respond in trials without a change in orientation. Attention was cued with a peripheral visual precue that indicated the likely location of an orientation change. Speed et al. (2020) used a probability manipulation, although of a different kind than in this study. They trained mice to detect gratings of varying contrast that were presented at one of two possible locations. The grating location was kept constant during blocks of approximately 25 trials, and the important observation was that detection performance improved during these blocks. This improvement in accuracy was attributed to attention, which was suggested to shift gradually, across a larger number of trials toward the fixed stimulus location. In our study, the effects of attention were only expressed on trials with long RTs. It is therefore of interest that the average RT in our study was shorter than that in Speed et al. (2020) and Wang and Krauzlis (2018), although the differences between paradigms complicate direct comparisons between RTs. For example, the mice of the study by Speed et al. (2020) were on less strict fluid restriction regime, whereas Wang and Krauzlis (2018) used a food reward. Nevertheless, these results, taken together, support our conjecture that differences in spatial attention (and stimulus-response compatibility; see below) between humans and mice expressed in our task may be explained by distinct strategies.

Previous studies suggest that the orientation discrimination threshold in mice is around $15-20^{\circ}$ (Jin, Beck, \& Glickfeld, 2019; Resulaj, Ruediger, Olsen, \& Scanziani, 2018; Aoki, Tsubota, Goya, \& Benucci, 2017). The stimuli used in our mouse task were high-contrast, orthogonal gratings, suggesting that the task should have been easy. One may therefore ask if the performance might have been at ceiling, leaving little room for spatial attention effects. However, this seems unlikely for two reasons. First, Posner (1980) demonstrated that spatial attention can have beneficial effects in very simple perceptual tasks. Second, the performance of our mice was not at ceiling. It was lower than might have been expected based on previous orientation discrimination studies. The lower accuracy was presumably caused by the mice favoring a strategy with short RTs, which is susceptible to stimulus-response compatibility effects, provokes errors, and is less susceptible to spatial attention effects (Speed et al., 2020; Wang \& Krauzlis, 2018). Further research is required to determine how spatial attention, stimulus-response compatibility, and accuracy interact in mice. The use of a licking response may also have contributed, because it may be more prone to impulsive responses than other response modalities, such as running/stopping on a treadmill, the turning of a wheel, or the release of a lever, which were used in previous studies. As a result, the accuracy of the mice was not at ceiling, leaving room for spatial attention effects, but these effects only occurred on trials with long RTs.

\section{Stimulus-Response Compatibility (Simon) Effect}

We found a very strong effect of stimulus-response compatibility on the accuracy of mice and a weaker effect in humans, which only occurred on trials with short RTs. Although the classical Simon effect was demonstrated as 
a shorter RT for compatible than incompatible stimulusresponse combinations (Simon, 1969), it can also influence the subject's accuracy (Craft \& Simon, 1970). Here, we only found an effect on accuracy. What prevents the mouse from reporting incompatible stimulus-response combinations? Previous studies proposed that the effect is caused by a conflict between two cognitive processes: a dorsal stream process planning a response toward the side of the stimulus and a ventral stream process implementing the desired stimulus-response mapping of orientations onto a left or right response (Salzer et al., 2017; De Jong et al., 1994). Our results revealed that mice experience severe difficulty in selecting a response opposite to the side of the stimulus. In other words, the proposed dorsal stream process may dominate the ventral stream process. Indeed, compared to primates, in the mouse, there are fewer hierarchical processing levels between visual cortex and motor structures not only via cortical routes but also via subcortical routes, including the superior colliculus thalamus and basal ganglia (Hu et al., 2019; Gămănuţ et al., 2018; Khibnik, Tritsch, \& Sabatini, 2014; Saint-Cyr, Ungerleider, \& Desimone, 1990), which could lead to a biased propagation of neuronal activity toward motor structures coding for a response to the same side as the stimulus. Future studies could directly test this hypothesis by shifting the balance between these processing streams, for example, using optogenetics (Guo et al., 2014).

In addition, there may have been differences in the motivation to respond quickly in the task between species. Mice were motivated through a fluid restriction regime, whereas human participants were rewarded with a payment that was not tied to performance at the end of the task. This may have led to rushed responses in mice, which aim to obtain fluid rewards as quickly as possible.

To conclude, mice employ a strategy in an orientation discrimination task in which they respond faster than human participants do. Stimulus-response compatibility effects are stronger in mice, whereas the effect of visual attention is only present in the subset of trials with the longest RTs. Human participants respond slower and have smaller stimulus-response effects, and the effects of attention shift are pronounced. However, when we only regard trials with very fast responses in humans, their performance exhibits a larger resemblance to that of mice. Our results not only agree with previous studies that mice are a valuable model to study neural correlates of response selection (Odoemene, Pisupati, Nguyen, \& Churchland, 2018) but also indicate that care is required when selecting paradigms to examine endogenous attention shifts, because some useful paradigms for humans do not readily translate to the mouse.

\section{Acknowledgments}

We thank the animal caretakers at the Netherlands Institute of Neuroscience for their assistance. We thank Birte Forstmann for her valuable comments on the paper. We thank the two anonymous reviewers for their very insightful comments, which helped improve our paper. This work was supported by the Dutch Research Council (NWO) (Earth and Life Sciences [ALW] grant 823-02-010), the European Union's Horizon 2020 and FP7 Research and Innovation Program (grant agreements 720270 and 785907 "Human Brain Project SGA1 and SGA2," ERC grant agreement 339490 "Cortic_al_gorithms," Amsterdam Neuroscience Alliance Project [CIA-2019-01]), and the Friends Foundation of the Netherlands Institute for Neuroscience.

Reprint requests should be sent to Pieter R. Roelfsema, Netherlands Institute for Neuroscience, Meibergdreef 47, Amsterdam, NoordHolland 1105 BA, the Netherlands, or via e-mail: p.roelfsema @nin.knaw.nl.

\section{Funding Information}

Amsterdam Neuroscience, grant number: Amsterdam Neuroscience Alliance Project (CIA-2019). H2020 European Research Council (http://dx.doi.org/10.13039/100010663), grant number: 339490 "Cortic_al_gorithms". Horizon 2020 Framework Programme (http://dx.doi.org/10.13039 /100010661), grant number: agreements 720270 \& 785907 "HBP SGA1 and SGA2". Friends Foundation of the Netherlands Institute for Neuroscience. FP7 Ideas: European Research Council (http://dx.doi.org/10.13039/100011199), grant number: agreements 720270 and 785907 "HBP SGA1 and SGA2". Nederlandse Organisatie voor Wetenschappelijk Onderzoek (http://dx.doi.org/10.13039/501100003246), grant number: ALW grant 823-02-010.

\section{Diversity in Citation Practices}

A retrospective analysis of the citations in every article published in this journal from 2010 to 2020 has revealed a persistent pattern of gender imbalance: Although the proportions of authorship teams (categorized by estimated gender identification of first author/last author) publishing in the Journal of Cognitive Neuroscience (JoCN) during this period were $\mathrm{M}(\mathrm{an}) / \mathrm{M}=.408, \mathrm{~W}(\mathrm{oman}) / \mathrm{M}=.335$, $\mathrm{M} / \mathrm{W}=.108$, and $\mathrm{W} / \mathrm{W}=.149$, the comparable proportions for the articles that these authorship teams cited were $\mathrm{M} / \mathrm{M}=.579, \mathrm{~W} / \mathrm{M}=.243, \mathrm{M} / \mathrm{W}=.102$, and $\mathrm{W} / \mathrm{W}=.076$ (Fulvio et al., JoCN, 33:1, pp. 3-7). Consequently, JoCN encourages all authors to consider gender balance explicitly when selecting which articles to cite and gives them the opportunity to report their article's gender citation balance.

\section{REFERENCES}

Adesnik, H., Bruns, W., Taniguchi, H., Huang, Z. J., \& Scanziani, M. (2012). A neural circuit for spatial summation in visual cortex. Nature, 490, 226-231. DOI: https://doi.org/10.1038 /nature11526, PMID: 23060193, PMCID: PMC3621107

Aoki, R., Tsubota, T., Goya, Y., \& Benucci, A. (2017). An automated platform for high-throughput mouse behavior and physiology with voluntary head-fixation. Nature Communications, 8, 1196. DOI: https://doi.org/10.1038/s41467-017-01371-0, PMID: 29084948, PMCID: PMC5662625

Arrington, C. M., Carr, T. H., Mayer, A. R., \& Rao, S. M. (2000). Neural mechanisms of visual attention: Object-based selection 
of a region in space. Journal of Cognitive Neuroscience. DOI: https://doi.org/10.1162/089892900563975, PMID: 11506651

Bisley, J. W., \& Goldberg, M. E. (2003). Neuronal activity in the lateral intraparietal area and spatial attention. Science, 299, 81-86. DOI: https://doi.org/10.1126/science.1077395, PMID: 12511644

Bleckert, A., Schwartz, G. W., Turner, M. H., Rieke, F., \& Wong, R. O. L. (2014). Visual space is represented by nonmatching topographies of distinct mouse retinal ganglion cell types. Current Biology, 24, 310-315. DOI: https://doi.org/10.1016 j.cub.2013.12.020, PMID: 24440397, PMCID: PMC3990865

Bushnell, M. C., Goldberg, M. E., \& Robinson, D. L. (1981). Behavioral enhancement of visual responses in monkey cerebral cortex. I. Modulation in posterior parietal cortex related to selective visual attention. Journal of Neurophysiology, 46, 755-772. DOI: https://doi.org/10.1152/jn.1981.46.4.755, PMID: 7288463

Bushnell, P. J. (1995). Overt orienting in the rat: Parametric studies of cued detection of visual targets. Behavioral Neuroscience, 109, 1095-1105. DOI: https://doi.org/10.1037 /0735-7044.109.6.1095, PMID: 8748960

Carandini, M., \& Churchland, A. K. (2013). Probing perceptual decisions in rodents. Nature Neuroscience, 16, 824-831. DOI: https://doi.org/10.1038/nn.3410, PMID: 23799475, PMCID: PMC 4105200

Corbetta, M., Shulman, G. L., Miezin, F. M., \& Petersen, S. E. (1995). Superior parietal cortex activation during spatial attention shifts and visual feature conjunction. Science, 270, 802-805. DOI: https://doi.org/10.1126/science.270.5237.802, PMID: 7481770

Courtière, A., Hardouin, J., Burle, B., Vidal, F., \& Hasbroucq, T. (2007). Simon effect in the rat: A new model for studying the neural bases of the dual-route architecture. Behavioural Brain Research, 179, 69-75. DOI: https://doi.org/10.1016 /j.bbr.2007.01.012, PMID: 17313980

Craft, J. L., \& Simon, J. R. (1970). Processing symbolic information from a visual display: Interference from an irrelevant directional cue. Journal of Experimental Psychology, 83, 415-420. DOI: https://doi.org/10.1037/h0028843, PMID: 4098174

De Jong, R., Liang, C. C., \& Lauber, E. (1994). Conditional and unconditional automaticity: A dual route process model of effects of spatial stimulus-response correspondence. Journal of Experimental Psychology: Human Perception and Performance, 20, 731-750. DOI: https://doi.org/10.1037 /0096-1523.20.4.731, PMID: 8083631

Gămănuţ, R., Kennedy, H., Toroczkai, Z., Ercsey-Ravasz, M., Van Essen, D. C., Knoblauch, K., et al. (2018). The mouse cortical connectome, characterized by an ultra-dense cortical graph, maintains specificity by distinct connectivity profiles. Neuron, 97, 698-715. DOI: https://doi.org/10.1016 /j.neuron.2017.12.037, PMID: 29420935, PMCID: PMC5958229

Gandhi, S. P., Heeger, D. J., \& Boynton, G. M. (1999). Spatial attention affects brain activity in human primary visual cortex. Proceedings of the National Academy of Sciences, U.S.A., 96, 3314-3319. DOI: https://doi.org/10.1073/pnas.96.6.3314, PMID: 10077681, PMCID: PMC15939

Gottlieb, J. P., Kusunoki, M., \& Goldberg, M. E. (1998). The representation of visual salience in monkey parietal cortex. Nature, 391, 481-484. DOI: https://doi.org/10.1038/35135, PMID: 9461214

Guo, Z., Hires, S., Li, N., O'Connor, D., Komiyama, T., Ophir, E., et al. (2014). Procedures for behavioral experiments in head-fixed mice. PLoS One, 9, e88678. DOI: https:/doi .org/10.1371/journal.pone.0088678, PMID: 24520413, PMCID: PMC3919818

Herrero, J. L., Gieselmann, M. A., Sanayei, M., \& Thiele, A. (2013). Attention-induced variance and noise correlation reduction in macaque $\mathrm{v} 1$ is mediated by NMDA receptors. Neuron, 78, 729-739. DOI: https://doi.org/10.1016/j.neuron 2013.03.029, PMID: 23719166, PMCID: PMC3748348

Herrington, T. M., \& Assad, J. A. (2009). Neural activity in the middle temporal area and lateral intraparietal area during endogenously cued shifts of attention. Journal of Neuroscience, 29, 14160-14176. DOI: https://doi.org/10.1523/JNEUROSCI .1916-09.2009, PMID: 19906965 , PMCID: PMC2913246

Hu, F., Kamigaki, T., Zhang, Z., Zhang, S., Dan, U., \& Dan, Y. (2019). Prefrontal corticotectal neurons enhance visual processing through the superior colliculus and pulvinar thalamus. Neuron, 104, 1141-1152. DOI: https://doi.org /10.1016/j.neuron.2019.09.019, PMID: 31668485

Jin, M., Beck, J. M., \& Glickfeld, L. L. (2019). Neuronal adaptation reveals a suboptimal decoding of orientation tuned populations in the mouse visual cortex. Journal of Neuroscience, 39, 3867-3881. DOI: https://doi.org/10.1523/JNEUROSCI.3172 -18.2019, PMID: 30833509, PMCID: PMC6520502

Katzner, S., \& Weigelt, S. (2013). Visual cortical networks: Of mice and men. Current Opinion in Neurobiology, 23, 202-206. DOI: https://doi.org/10.1016/j.conb.2013.01.019, PMID: 23415830

Khibnik, L. A., Tritsch, N. X., \& Sabatini, B. L. (2014). A direct projection from mouse primary visual cortex to dorsomedial striatum. PLoS One, 9, e104501. DOI: https://doi.org/10 .1371/journal.pone.0104501, PMID: 25141172, PMCID: PMC4139305

Lu, C., \& Proctor, R. W. (1995). The influence of irrelevant location information on performance: A review of the Simon and spatial Stroop effects. Psychonomic Bulletin \& Review, 2, 174-207. DOI: https://doi.org/10.3758/BF03210959, PMID: 24203654

Müller, M. M., Teder-Sälejärvi, W., \& Hillyard, S. A. (1998). The time course of cortical facilitation during cued shifts of spatial attention. Nature Neuroscience, 1, 631-634. DOI: https:// doi.org/10.1038/2865, PMID: 10196572

Nandy, A. S., Nassi, J. J., \& Reynolds, J. H. (2017). Laminar organization of attentional modulation in macaque visual area V4. Neuron, 93, 235-246. DOI: https://doi.org/10.1016/j.neuron .2016.11.029, PMID: 27989456, PMCID: PMC5217483

Niell, C. M., \& Stryker, M. P. (2008). Highly selective receptive fields in mouse visual cortex. Journal of Neuroscience, 28, 7520-7536. DOI: https://doi.org/10.1523/JNEUROSCI.0623 -08.2008, PMID: 18650330, PMCID: PMC3040721

Odoemene, O., Pisupati, S., Nguyen, H., \& Churchland, A. K. (2018). Visual evidence accumulation guides decision-making in unrestrained mice. Journal of Neuroscience, 38, 10143-10155. DOI: https://doi.org/10.1523/JNEUROSCI.3478-17.2018, PMID: 30322902, PMCID: PMC6246883

Payne, H. L., \& Raymond, J. L. (2017). Magnetic eye tracking in mice. eLife, 6, e29222. DOI: https://doi.org/10.7554/eLife 29222, PMID: 28872455, PMCID: PMC5584990

Poort, J., Raudies, F., Wannig, A., Lamme, V. A. F., Neumann, H., \& Roelfsema, P. R. (2012). The role of attention in figureground segregation in areas V1 and V4 of the visual cortex. Neuron, 75, 143-156. DOI: https://doi.org/10.1016/j.neuron .2012.04.032, PMID: 22794268

Posner, M. I. (1980). Orienting of attention. Quarterly Journal of Experimental Psychology, 32, 3-25. DOI: https://doi.org 10.1080/00335558008248231, PMID: 7367577

Resulaj, A., Ruediger, S., Olsen, S. R., \& Scanziani, M. (2018). First spikes in visual cortex enable perceptual discrimination. eLife, 7, e34044. DOI: https://doi.org/10.7554/eLife.34044, PMID: 29659352, PMCID: PMC5902162

Reynolds, J. H., \& Chelazzi, L. (2004). Attentional modulation of visual processing. Annual Review of Neuroscience, 27, 611-647. DOI: https://doi.org/10.1146/annurev.neuro.26.041002.131039, PMID: 15217345 
Ridderinkhof, K. R., Scheres, A., Oosterlaan, J., \& Sergeant, J. A (2005). Delta plots in the study of individual differences: New tools reveal response inhibition deficits in $\mathrm{AD} / \mathrm{HD}$ that are eliminated by methylphenidate treatment. Journal of Abnormal Psychology, 114, 197-215. DOI: https://doi.org /10.1037/0021-843X.114.2.197, PMID: 15869351

Roelfsema, P. R., Lamme, V. A. F., \& Spekreijse, H. (1998). Object-based attention in the primary visual cortex of the macaque monkey. Nature, 395, 376-381. DOI: https://doi .org/10.1038/26475, PMID: 9759726

Rosen, A. C., Rao, S. M., Caffarra, P., Scaglioni, A., Bobholz, J. A., Woodley, S. J., et al. (1999). Neural basis of endogenous and exogenous spatial orienting: A functional MRI study. Journal of Cognitive Neuroscience, 11, 135-152. DOI: https://doi .org/10.1162/089892999563283, PMID: 10198130

Sachidhanandam, S., Sreenivasan, V., Kyriakatos, A., Kremer, Y., \& Petersen, C. C. H. (2013). Membrane potential correlates of sensory perception in mouse barrel cortex. Nature Neuroscience, 16, 1671-1677. DOI: https://doi.org/10.1038 /nn.3532, PMID: 24097038

Saint-Cyr, J. A., Ungerleider, L. G., \& Desimone, R. (1990). Organization of visual cortical inputs to the striatum and subsequent outputs to the pallido-nigral complex in the monkey. Journal of Comparative Neurology, 298, 129-156. DOI: https://doi.org/10.1002/cne.902980202, PMID: 1698830

Sakatani, T., \& Isa, T. (2007). Quantitative analysis of spontaneous saccade-like rapid eye movements in $\mathrm{C} 57 \mathrm{BL} / 6$ mice. Neuroscience Research, 58, 324-331. DOI: https://doi .org/10.1016/j.neures.2007.04.003, PMID: 17482700

Salzer, Y., de Hollander, G., \& Forstmann, B. U. (2017). Sensory neural pathways revisited to unravel the temporal dynamics of the Simon effect: A model-based cognitive neuroscience approach. Neuroscience \& Biobehavioral Reviews, 77, 48-57. DOI: https://doi.org/10.1016/j.neubiorev.2017.02.023, PMID: 28238943

Schall, J. D., \& Hanes, D. P. (1993). Neural basis of saccade target selection in frontal eye field during visual search. Nature, 366 , 467-469. DOI: https://doi.org/10.1038/366467a0, PMID: 8247155

Schall, J. D., \& Thompson, K. G. (1999). Neural mechanisms of selection and control of visually guided eye movements. Annual Review of Neuroscience, 22, 241-259. DOI: https:// doi.org/10.1146/annurev.neuro.22.1.241, PMID: 10202539

Self, M. W., Lorteije, J. A. M., Vangeneugden, J., van Beest, E. H., Grigore, M. E., Levelt, C. N., et al. (2014). Orientation-tuned surround suppression in mouse visual cortex. Journal of Neuroscience, 34, 9290-9304. DOI: https://doi.org/10.1523 /JNEUROSCI.5051-13.2014, PMID: 25009262, PMCID: PMC6608354

Self, M. W., Peters, J. C., Possel, J. K., Reithler, J., Goebel, R., Ris, P., et al. (2016). The effects of context and attention on spiking activity in human early visual cortex. PLoS Biology, 14, e1002420. DOI: https://doi.org/10.1371/journal.pbio.1002420, PMID: 27015604, PMCID: PMC 4807817

Shomstein, S., \& Behrmann, M. (2006). Cortical systems mediating visual attention to both objects and spatial locations. Proceedings of the National Academy of Sciences, U.S.A., 103, 11387-11392.
DOI: https://doi.org/10.1073/pnas.0601813103, PMID: 16840559, PMCID: PMC1544095

Shomstein, S., \& Yantis, S. (2004). Configural and contextual prioritization in object-based attention. Psychonomic Bulletin E Review, 11, 247-253. DOI: https://doi.org/10 .3758/BF03196566, PMID: 15260189

Simon, J. R. (1969). Reactions toward the source of stimulation. Journal of Experimental Psychology, 81, 174-176. DOI: https://doi.org/10.1037/h0027448, PMID: 5812172

Somers, D. C., Dale, A. M., Seiffert, A. E., \& Tootell, R. B. (1999). Functional MRI reveals spatially specific attentional modulation in human primary visual cortex. Proceedings of the National Academy of Sciences, U.S.A., 96, 1663-1668.

DOI: https://doi.org/10.1073/pnas.96.4.1663, PMID: 9990081, PMCID: PMC15552

Speed, A., Del Rosario, J., Mikail, N., \& Haider, B. (2020). Spatial attention enhances network, cellular and subthreshold responses in mouse visual cortex. Nature Communications, 11, 505. DOI: https://doi.org/10.1038/s41467-020-14355-4, PMID: 31980628, PMCID: PMC6981183

Sridharan, D., Ramamurthy, D. L., Schwarz, J. S., \& Knudsen, E. I. (2014). Visuospatial selective attention in chickens. Proceedings of the National Academy of Sciences, U.S.A., 111, E2056-E2065. DOI: https://doi.org/10.1073/pnas.1316824111, PMID: 24753566, PMCID: PMC4024881

Steinmetz, N. A., Zatka-Haas, P., Carandini, M., \& Harris, K. D. (2019). Distributed coding of choice, action and engagement across the mouse brain. Nature, 576, 266-273. DOI: https:// doi.org/10.1038/s41586-019-1787-x, PMID: 31776518 , PMCID: PMC6913580

Vaiceliunaite, A., Erisken, S., Franzen, F., Katzner, S., \& Busse, L. (2013). Spatial integration in mouse primary visual cortex

Journal of Neurophysiology, 110, 964-972. DOI: https://doi .org/10.1152/jn.00138.2013, PMID: 23719206, PMCID: PMC3742980

Van Den Bergh, G., Zhang, B., Arckens, L., \& Chino, Y. M. (2010). Receptive-field properties of V1 and V2 neurons in mice and macaque monkeys. Journal of Comparative Neurology, 518, 2051-2070. DOI: https://doi.org/10.1002 /cne.22321, PMID: 20394058, PMCID: PMC2881339

Wang, F., Chen, M., Yan, Y., Zhaoping, L., \& Li, W. (2015). Modulation of neuronal responses by exogenous attention in macaque primary visual cortex. Journal of Neuroscience, 35 , 13419-13429. DOI: https://doi.org/10.1523/JNEUROSCI .0527-15.2015, PMID: 26424888, PMCID: PMC6605478

Wang, L., \& Krauzlis, R. J. (2018). Visual selective attention in mice. Current Biology, 28, 676-685. DOI: https://doi .org/10.1016/j.cub.2018.01.038, PMID: 29456140, PMCID: PMC5914527

Wang, Q., \& Burkhalter, A. (2007). Area map of mouse visual cortex. Journal of Comparative Neurology, 357, 339-357. DOI: https://doi.org/10.1002/cne.21286, PMID: 17366604

Wang, Q., Gao, E., \& Burkhalter, A. (2011). Gateways of ventral and dorsal streams in mouse visual cortex. Journal of Neuroscience, 31, 1905-1918. DOI: https://doi.org/10.1523 /JNEUROSCI.3488-10.2011, PMID: 21289200, PMCID: PMC3040111 